\title{
Yolk Sac
}

National Cancer Institute

\section{Source}

National Cancer Institute. Yolk Sac. NCI Thesaurus. Code C14128.

Membranous sac on the ventral aspect of the developing embryo that acts as a primitive circulatory system as well as providing nourishment. 